\title{
High-Sensitive Cardiac Troponin I - An Important Biomarker in the Course of COVID-19 Disease in Adult Patients
}

\author{
Mariana Georgieva Yordanova ${ }^{1,2 *}$ \\ ${ }^{1}$ Military Medical Academy, Multiprofile Hospital for Active Treatment, Bulgaria \\ ${ }^{2}$ Department of and Clinical Laboratory, Medical University of Varna, Bulgaria
}

*Corresponding author: Mariana Georgieva Yordanova, Military Medical Academy, Multiprofile Hospital for Active Treatment, Varna, Bulgaria.
Received Date: November 15, 2021

Published Date: December 01, 2021

\begin{abstract}
Introduction: SARS-CoV-2 virus, in addition to causing pneumonia, can cause direct damage to the heart, inducing myocarditis, pericarditis or AMI with significant impairment of cardiac contractility. Adult patients with hypertension, diabetes mellitus and obesity are at increased cardiovascular risk and complications from COVID-19.
\end{abstract}

Aim: To assess high-sensitivity troponin I (hs-TnI) levels among hospitalized patients with COVID-19 as a biomarker associated with myocardial damage and determine the correlation with disease severity and outcome.

Material and Method: A retrospective analysis of hospitalized patients was performed between January 1 and May 1, 2021, with clinical and laboratory evidence of COVID-19. Monitored were the laboratory parameters of myocardial damage with baseline and peak hs-TnI values above the median ( $\geq 19.8 \mathrm{ng} / \mathrm{L}$ ) in 110 patients. The extent of the inflammatory response (CRP), coagulation status (DDimer), acid-base balance parameters, hypoxemia abnormalities and LDH levels according to disease severity and outcome, and length of hospital stay was assessed. Data were evaluated using descriptive, correlation and multivariate analysis.

Results: The present study showed a statistically significant relationship between disease severity and heart damage. The hs-TnI values in patients with COVID-19 and CVD were statistically significantly higher than those with COVID-19 but without CVD. The most significant levels of the cardiac biomarker are observed in deceased patients.

Conclusion: High-sensitive troponin is a valuable biomarker for early diagnosis and assessment of disease progression with a worse prognosis in patients with COVID-19. Elevated hs-TnI in patients with SARS-CoV-2 infection who have concomitant heart disease may suggest the severity of the clinical picture and the course of the disease.

\section{Introduction}

From early 2020, the world faces a new medical challenge, a pandemic caused by an unknown SARS-CoV-2 coronavirus, requiring new knowledge and approaches. Today, more than 250 million people worldwide are affected by the covid infection, of whom $2.1 \%$ have lost their lives. The most common pathological manifestation of SARS-CoV-2 is respiratory distress syndrome, considered the leading cause of death. However, with each new epidemic wave, more evidence also accumulates of direct damage to the heart, causing myocarditis, arrhythmia, and acute coronary syndrome as likely concomitant damage. The presence of previous cardiovascular disease or cardiovascular risk factors leads to a higher mortality rate than patients without prior cardiovascular disease [1]. In addition, several studies have shown that COVID-19 patients who did not have heart disease before infection may also develop cardiac complications [2].

Cardiac troponins are biomarkers that have gained a reputation as the gold standard in diagnosing myocardial damage. The troponin complex consists of three subunits (troponin C, troponin 
$\mathrm{T}$ and troponin I). Together with calcium ions, troponin proteins regulate and facilitate the interaction between actin and myosin fibres during muscle contraction, including the myocardium. Troponin I is particular for the heart muscle and is not isolated from skeletal muscle. This absolute specificity makes it an ideal marker for myocardial damage [3].

Cardiac isoforms of troponins are quantitative markers of cardiomyocyte damage, and the likelihood of AMI increases with increasing levels in the blood. Their increase is observed in the interval 4-8 hours after myocardial injury, with a peak value of 12-24 hours and retention in the levels for 7-10 days [4]. A Contemporary high-sensitivity cardiac troponin (hs-cTn) assays can detect concentrations 10 to 100 times lower than conventional assays, increasing their diagnostic accuracy in patients with acute chest pain [5]. In this aspect, it is reasonable to investigate hs-TnI in patients with COVID-19 as an essential biomarker for both an early diagnosis of myocardial injury and prognosis.

\section{Aim}

To analyze the levels of high-sensitivity troponin I among hospitalized patients with COVID-19 as a biomarker associated with myocardial injury and to determine the correlation with disease severity and outcome.

\section{Material and Method}

112 patients from 320 hospitalized patients with proven SARSCoV-2 infection in the period 1.02. to 1.05 .2021 were found to have cardiac lesions with initial and peak hs-TnI values above the median (URL of 99 th percentile $\geq 19.8 \mathrm{ng} / \mathrm{L}$ ). The review included
82 men and 30 women aged $67.7 \pm 15.3$ years and $71.6 \pm 18.8$ years, respectively. Levels of hs-TnI were determined by chemiluminescent immunoassay on an Assay 2 analyzer (B. Coulter - USA). The extent of inflammatory response was assessed by CRP values (Olympus AU640 B. Coulter - USA), change in coagulation status and tendency to thrombogenicity (DDimer - Stago Satelite), disturbances in $\mathrm{AB}$ balance and hypoxemia by KGA Modular Pro (ESCHWEILER Germany), LDH levels (Olympus AU640 B. Coulter - USA), length of stay and disease outcome. The severity of SARS-CoV-2 infection was assessed according to WHO criteria from January 2021.

Data analysis was done using GraphPad Prism v. 6.0 software by standard statistical methods (descriptive statistics, Anova with Bonferroni correction for median comparison, and Spearman correlation analysis). Biochemical parametric data were presented as mean \pm standard deviation. Statistical significance was indicated at $\mathrm{p}<0.05$.

\section{Result}

According to the WHO criteria for the clinical course, patients were divided into groups - moderate grade $(22 \%)$, severe grade (45\%) and critical grade (33\%). Eighteen deaths with critical grade disease, representing $16.1 \%$ (12 males and six females) were. Of these, only two men in young age (45-50 years) had no evidence of previous and concomitant diseases. All other patients had comorbidities, most commonly $\mathrm{AH}$ alone or combined with CVD and/or diabetes and obesity. Furthermore, COVID-19 patients according to clinical presentation (subjective and objective criteria) of acute cardiac injury were divided into three groups (Table 1 ).

Table 1: Parameters were studied in COVID-19 patients in the three groups. Patients with CVD events, those who died as a result of them and patients with COVID-19 without CVD disorders.

\begin{tabular}{|c|c|c|c|c|}
\hline Parameters & Patients with CVD & Patients without CVD & Died & $p$ value \\
\hline hs-TnI [ng/L] & $\begin{array}{c}1258 \\
\text { (IQR 98- 3580) }\end{array}$ & $\begin{array}{c}8.694 \\
\text { (IQR 6.12- 12.3) }\end{array}$ & $\begin{array}{c}4996 \\
\text { (IQR 818-7527) }\end{array}$ & $\begin{array}{l}\mathrm{p}^{*}<0.001 \\
\mathrm{p}^{* *}<0.001\end{array}$ \\
\hline $\mathrm{CRP}[\mathrm{mg} / \mathrm{L}]$ & $\begin{array}{c}172.0 \\
\text { (IQR 122- 222) }\end{array}$ & $\begin{array}{c}147.4 \\
\text { (IQR102-276) }\end{array}$ & $\begin{array}{c}168.5 \\
\text { (IQR } 90-226)\end{array}$ & $\begin{array}{l}*_{\mathrm{ns}} \\
*_{\mathrm{ns}}\end{array}$ \\
\hline $\operatorname{DDimer}[\mu \mathrm{g} / \mathrm{ml}]$ & $\begin{array}{c}6.5 \\
\text { (IQR 3.2-17.8) }\end{array}$ & $\begin{array}{c}4.9 \\
\text { (IQR 2.6-11.4) }\end{array}$ & $\begin{array}{c}8.2 \\
\text { (IQR 3.1-13.8) }\end{array}$ & $\begin{array}{c}*_{\mathrm{ns}} \\
{ }_{\mathrm{*}} \mathrm{p}=0.058\end{array}$ \\
\hline $\mathrm{LDH}[\mathrm{U} / \mathrm{L}]$ & $\begin{array}{c}487.7 \\
\text { (IQR 334-567) }\end{array}$ & $\begin{array}{c}460 \\
\text { (IQR340-588) }\end{array}$ & $\begin{array}{c}668 \\
\text { (IQR 485-788 }\end{array}$ & $\begin{array}{c}*_{\mathrm{ns}} \\
\mathrm{p}^{* *}<0.01\end{array}$ \\
\hline $\mathrm{sO}_{2}[\%]$ & $\begin{array}{c}82.9 \% \\
\text { (IQR78.3-87.2) }\end{array}$ & $\begin{array}{c}85.5 \% \\
\text { (IQR81.3-88.2) }\end{array}$ & $\begin{array}{c}83.5 \% \\
\text { (IQR70-86.5) }\end{array}$ & $\begin{array}{l}* n s \\
* * n s\end{array}$ \\
\hline
\end{tabular}

*The comparative analysis of the differences between the three studied groups was performed with One way ANOVA with Bonferroni correction. ( $p$ * - comparison between patients with covid infection with CVD and those with covid but without CVD; $p^{* *}$ - comparison between the general group of patients with the Covid disease and CVD compared to those who died as a result of a heart attack.

The analysis performed found that patients with COVID-19 and cardiovascular symptoms or complications had significantly higher hs-TnI values than other patients with COVID-19 but without cardiac problems. The highest myocardial necrosis marker values were found in those who died due to AMI, myocarditis, rhythm and conduction disturbances, or PTE. In the group with lethal outcome, hs-TnI values showed a statistically significant difference compared to the entire CVD group and those without such complications $(\mathrm{p}<0.001)$. Regarding the other studied parameters characterizing the severity of pulmonary perfusion disturbance, haemostasis and hypoxaemia, no such differences were found between groups. There was a tendency for a difference in DDimer values in the deceased patients compared to the general CVD group. Only LDH as an enzyme reflecting both necroses and activated anaerobic glycolysis showed significantly higher levels in the deceased than the total patient group with CVD complications. This explains the moderate 
statistically significant correlation between hs-TnI and LDH values in the overall COVID-19 group with CVD ( $\mathrm{r}=0.438 \mathrm{p}=0.0008)$.

We found that hs-TnI levels showed a high correlation with disease severity (Figure 1). A significant degree of correlation was observed between the severity of the disease and the length of hospital stay ( $r=0.635 ; p<0.001)$. The mean length of stay in patients with CVD was 16 days while in the critical course of the disease and lethal outcome is 14 days (range 8-30 days). An analysis of the correlations between hs-TnI and other indicators for monitoring vital and metabolic disorders in patients from the group of the deceased revealed the following data (Table 2).

Table 2: Correlation between the values of hsTrl with the other studied indicators in deceased patients with Covid 19 and CVD incidents.

\begin{tabular}{|c|c|c|}
\hline Correlation dependencies & Correlation coefficient (r) \\
\hline hsTrI vs.CRP & 0.5298 & 0.014 \\
\hline hsTrI vs.DD & 0.2564 \\
\hline hsTrI vs. sO2 & -0.3023 \\
\hline hsTrI vs.LDH & 0.5186 & ns \\
\hline
\end{tabular}

*Nonparametric correlation analysis and calculated Spearman r coefficients were applied.

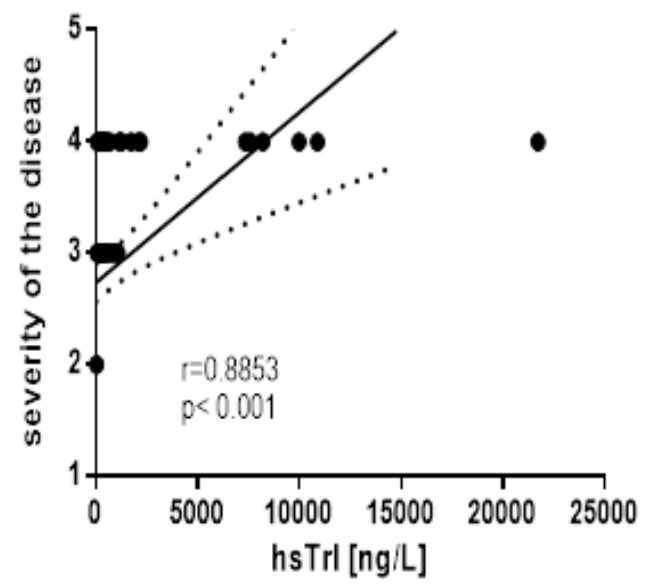

Figure 1: Correlation between hs-Tnl values and disease severity in patients with COVID-19 and CVD.

A moderate correlation between hs-TnI and LDH is currently established in the general group of COVID-19 and GCC. In the deceased group, this correlation is preserved and is significant in size (Figure 2B). We observed a similar correlation force with CRP, a biomarker of inflammation (Figure 2A). This is probably because $\mathrm{CRP}$, in addition to being a well-known acute phase protein, is a marker that reflects and correlates with the size of the necrotic myocardial lesion.

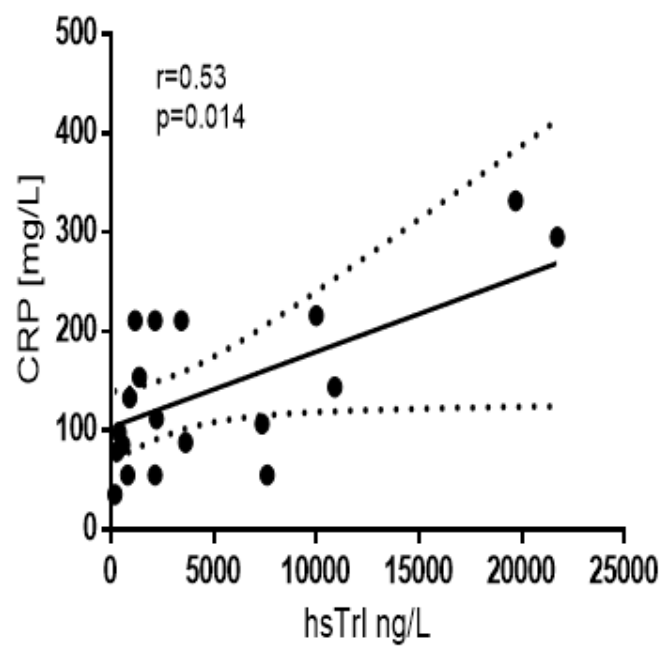

Figure 2A: Correlation between hs-Tnl and CRP values. 


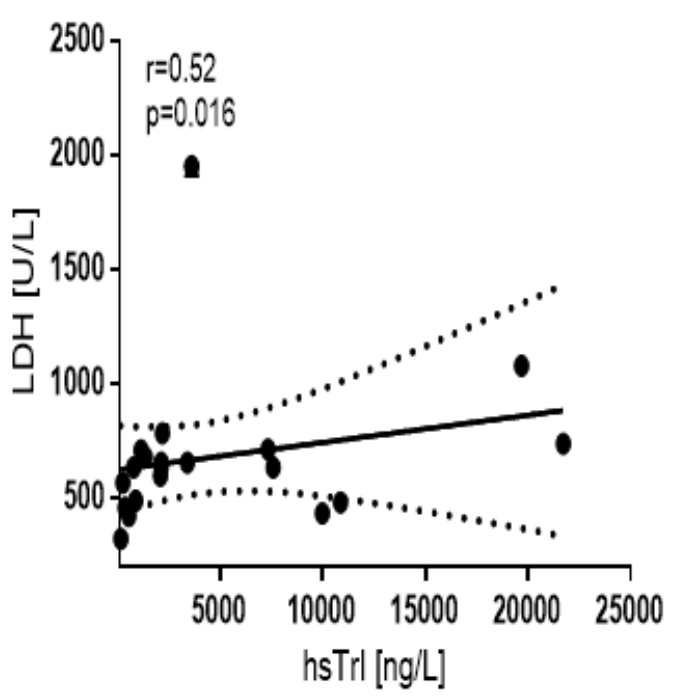

Figure 2B: Correlation between hs-Tnl and LDH values in deceased CVD patients.

\section{Discussion}

Shi et al. showed as early as 2020 that myocardial injury is a common condition among COVID-19 patients in Wuhan [6]. The authors reported that more than half of these patients died. A recent report suggests initial and follow-up testing during hospital stay of high-sensitivity troponin in patients with SARS-CoV-2 infection as a way to help clinicians identify individuals with possible cardiac damage and predict progression of COVID-19 to a fatal outcome [7]. The aetiology of acute myocardial injury in response to SARS-CoV-2 infection is still poorly understood. Possible mechanisms include cytokine storm resulting from a dysfunctional and uncontrolled immunologic response evidenced by elevated levels of d-dimer, IL6, CRP, and LDH and primary cardiac injury caused by SARS-CoV-2 [8]. One of the hypotheses is a direct attack on myocytes in the viraemic phase because of the affinity between the SARS-CoV Spike protein and angiotensin-converting enzyme type 2 (ACE-2), which is well represented in myocardial cells [9]. ACE-2 is also present in the vascular endothelium, so vasculitis and endothelial dysfunction are possible, further activating haemostasis with the occurrence of thromboembolic events and myocardial ischemic injury $[10,11]$.

The amount of ACE2 may vary between individuals as well as in different tissues and cells. Some evidence suggests that ACE2 may be higher in patients with hypertension, diabetes, and coronary artery disease, explaining their greater vulnerability to SARSCoV-2 infection [12]. The primary role of ACE2 is that of a negative regulator of the renin-angiotensin system (RAS) by degrading angiotensin II to angiotensin. With this, it exerts vasodilating, anti-inflammatory and antifibrotic effects by binding to the Mas receptor. The RAS plays a critical role in the maintenance of blood pressure homeostasis as well as water-electrolyte balance and is closely related to the pathophysiology of heart disease [13]

Our data show an increase in hs-TnI in patients with myocardial injury, consistent with the findings of the meta-analysis by Dawson Det al. [14]. There was also an increased risk of death with increasing cardiac marker levels found in other studies [15]. Patients with COVID-19 have reduced oxygen supply due to hypoxic respiratory failure and concomitant increased oxygen demand due to tachycardia, febrility and endocrine dysregulation, which enhances myocardial hypoxia [16]. Patients who passed through the Covid unit during the relevant period were predominantly elderly (over 65 years of age and $29.5 \%$ of them are over 80 years of age) with concomitant cardiovascular disease. This predisposed to SARS-CoV-2 induced myocardial injury and increased mortality associated with COVID-19. Ample evidence has now accumulated to explain the increased susceptibility and severity in the course of the disease, resulting from multiple factors, most commonly dependent on altered ACE2 expression, age, sex, medications and comorbidities s.

A clear picture of hypercoagulability with elevated D-dimer values is observed in the course of the disease. The most significantly increased levels of the parameter are seen in patients with critical stage disease. D-dimer is a fibrinolytic degradation product of pathologically increased blood clot formation. Some studies found that D-dimer levels at hospital admission predicted worse clinical outcomes [18]. In contrast to the meta-analysis by Dawson D, et al. in the present study, we found no statistically significant difference in DDimer between the group with and without CVD versus deceased [14]. COVID-19 infection likely causes a range of multidirectional pathological conditions in different patient groups, whereby differences between groups are blurred. However, the D-dimer is also an inflammatory marker of the acute phase, which may explain the non-statistical differences in the three groups of patients.

\section{Conclusion}

cTnI appears to be a beneficial biomarker, the gold standard for early diagnosis of cardiac complications and myocardial damage in the course of COVID-19 infection. Serum cTnI testing is an independent predictor of disease severity and mortality, especially in elderly patients with comorbidities. 


\section{Acknowledgement}

None.

\section{Conflict of Interest}

The author has no relevant affiliations or financial involvement with any organization or entity with a financial interest in or financial conflict with the subject matter or materials discussed in the manuscript.

\section{References}

1. Masataka Nishiga, Dao Wen Wang, Yaling Han, David B Lewis, Joseph C Wu (2020) COVID-19 and cardiovascular disease: from basic mechanisms to clinical perspectives. Nat Rev Cardiol 17: 543-558.

2. Indranill Basu-Ray, Nureddin $\mathrm{k}$ Almaddah, Adedayo Adeboye, Michael P Soos (2021) Cardiac Manifestations Of Coronavirus (COVID-19). In StatPearls [Internet]. Treasure Island (FL): StatPearls Publishing.

3. Mythili S, Malathi N (2015) Diagnostic markers of acute myocardial infarction. Biomed Rep 3(6): 743-748.

4. Anand Kumar (2018) Biomarkers in Acute Myocardial Infarction. National Journal of Basic Medical Sciences v. 8(3).

5. Pankaj Garg, Paul Morris, Asma Lina Fazlanie, Sethumadhavan Vijayan, Balazs Dancso, et al. (2017) Cardiac biomarkers of acute coronary syndrome: from history to high-sensitivity cardiac troponin. Intern Emerg Med 12(2): 147-155.

6. Shaobo Shi, Mu Qin, Bo Shen, Yuli Cai, Tao Liu, et al. (2020) Association of Cardiac Injury With Mortality in Hospitalized Patients With COVID-19 in Wuhan, China. JAMA Cardiol 5(7): 802-810.

7. Michela Salvatici, Barbara Barbieri, Sara Maria Giulia Cioffi, Emanuela Morenghi, Francesco Paolo Leone, et al. (2020) Association between cardiac troponin I and mortality in patients with COVID-19. Biomarkers 25(8): 634-640.

8. Savla SR, Prabhavalkar KS, Bhatt LK (2021) Cytokine storm associated coagulation complications in COVID-19 patients: Pathogenesis and Management. Expert Rev Anti Infect Ther 19(11): 1397-1413.
9. A Piccioni, M Brigida, V Loria, C Zanza, Y Longhitano, et al. (2020) Role of troponin in COVID-19 pandemic: a review of literature. Eur Rev Med Pharmacol Sci 24(19): 10293-10300.

10. Aldo Bonaventura, Alessandra Vecchié, Lorenzo Dagna, Kimberly Martinod, Dave L Dixon, et al. (2021) Endothelial dysfunction and immunothrombosis as key pathogenic mechanisms in COVID-19. Nat Rev Immunol 21: 319-329.

11. Simone Sala, Giovanni Peretto, Mario Gramegna, Anna Palmisano, Andrea Villatore, et al. (2020) Acute myocarditis presenting as a reverse TakoTsubo syndrome in a patient with SARS-CoV-2 respiratory infection. Eur Heart J 41(19): 1861-1862.

12. Wentao Ni, Xiuwen Yang, Deqing Yang, Jing Bao, Ran Li, et al. (2020) Role of angiotensin-converting enzyme 2 (ACE2) in COVID-19. Crit Care 24(1): 422.

13. Ciaglia E, Vecchione C, Puca AA (2020) COVID-19 Infection and Circulating ACE2 Levels: Protective Role in Women and Children. Front Pediatr 8: 206.

14. Dawson D, Dominic P, Sheth A, Modi M (2020) Prognostic value of Cardiac Biomarkers in COVID-19 Infection: A Meta-analysis. Preprint. Res Sq p.rs.3.rs-34729.

15. Khan S, Rasool ST, Ahmed SI (2021) Role of Cardiac Biomarkers in COVID-19: What Recent Investigations Tell Us. Curr Probl Cardiol 46(10): 100842

16. Gregorio Tersalvi, Marco Vicenzi, Davide Calabretta, Luigi Biasco, Giovanni Pedrazzini, et al. (2020) Elevated Troponin in Patients With Coronavirus Disease 2019: Possible Mechanisms. J Card Fail 26(6): 470475 .

17. Arno R Bourgonje, Amaal E Abdulle, Wim Timens, Jan-Luuk Hillebrands, Gerjan J Navis, et al. (2020) Angiotensin-converting enzyme 2 (ACE2), SARS-CoV-2 and the pathophysiology of coronavirus disease 2019 (COVID-19). J Pathol 251(3): 228-248.

18. Ning Tang, Dengju Li, Xiong Wang, Ziyong Sun (2020) Abnormal coagulation parameters are associated with poor prognosis in patients with novel coronavirus pneumonia. J Thromb Haemost 18: 844- 847. 